\title{
The Research and Application of Web Services in Enterprise Application Integration
}

\author{
Yan Cao, Yan Chen and Yiting Shen \\ College of Economics and Management, Dalian Maritime University, Dalian 116026, P.R. \\ China caoy123@163.com chenyan_dlmu@163.com atingle_cn@hotmail.com
}

\begin{abstract}
At present, most enterprises have a heterogeneous environment of legacy application, which lead to many "information isolated islands". Enterprises become more and more eager to share data in business processes over different systems. Traditional Enterprise Application Integration (EAI) methods usually integrate applications from peer to peer which has many defects. In This paper, a kind of EAI framework based on Web Services (WSEAI) has been put forward, which has nothing with any platform and programming language, so it can make the enterprise applications integrated together without gap. We have been successfully putting the EAI framework in one logistical company's WebGPS system and realized the integration based on Web Services.
\end{abstract}

Keywords: EAI, WSEAI, Web services, WebGPS system

\section{INTRODUCTION}

Text Enterprise Application Integration (EAI) refers to the seamless application systems integration of two or more enterprises by tying in the operation flow, application software, hardware and a variety of standards, so that the enterprises could work like one in business processing and information sharing. Traditional EAI uses distributed object technology-based point-to-point integration like RMI, CORBA, DCOM, etc., which usually could not meet the requirement of the enterprises and result in high cost and low efficiency. Therefore, a new solution is needed.

With the maturity of the Web Services technology, it has broken through the restriction of the traditional point-to-point application integration solution, and exerted great impact on the EAI. Considering current heterogeneous platforms, the main aim of the Web Services technology is to build a general-purpose technology level regardless of the platforms and languages, by which to realize the connection and integration [1].

In this Paper, based on the Web Services technology, we put forward the Web Services-based EAI Framework which is called as WSEAI, and its biggest difference between the traditional EAI solutions lays in its inter-enterprise integration, and at the same time its consideration of the interaction of the Web Services in different platforms, which could successfully integrate the legacy systems on Web Services base. The WSEAI make the integration of different application systems easier, cost- 
efficient and dynamically expandable, and even partial realization of the EAI will be very useful.

\section{DESIGN OF WSEAI}

Traditional EAI solution, for example, point-to-point integration or distributed object-based middleware integration, features with its standard processing of the interfaces of different information systems for integration, which lacks of flexibility and adaptability. But through standard Web protocols (HTTP, SMTP, etc.) and series standard protocols (XML, SOAP, WSDL, UDDI, etc), the standard Web Services technology has provided a new solution for the EAI.

Both the Intra-EAI and Inter-EAI have been taken into consideration in designing the Web Services-based EAI Framework.

As the basis of EAI, currently the Intra-EAI mainly needs to solve the communication problem of different modules. Different from the traditional EAI modules that use special protocol, by using the Web Services Technology, the modules' interfaces could be altered to standard Web Services interfaces before connecting with the external equipments. So by wrapping the interfaces modules of different intra-enterprise application systems through the Web Services technology, and publishing it to the private UDDI Registry, then the other application systems of the enterprise could realize its own function by invoking the Web Services. This could realize the communication of the scattered legacy systems, and consequently realize the Intra-EAI.

The virtual enterprise and Inter-EAI is one of the highlights in the future informatization. While by making use of the reuse mechanism of Web Services, there will be no need of external service programming, and the only requirement is to republish the Web Services to the public UDDI registry, and then the other enterprises could invoke the Web Services on the Internet, which could break through the regional restriction and realize the Inter-EAI.

Accordingly, the Web Services technology is very feasible to realize the EAI. In view of the need of the enterprise, in this Paper we designed the Web Services-based EAI Framework (WSEAI), and it is shown as Figure 1[1-2].

According to the Figure 1, the Web Services-based EAI Framework could be built on the platform of J2EE and NET, and the functions of its main modules are as follows:

(1) Web Services Adapter: Web Services adapter mainly alters the current application systems to the Web Services interface format and processes the binding invocation of the Web Services.

(2) SOAP Router: As key part of the integration framework, the SOAP router is used to transfer messages, its two main functions are: SOAP message router and message format conversion.

(3) Web Server: As the core of the integration framework, the main function of the Web Server is to provide interactive Web pages and transmit the request of the upper level to SOAP router, and then communicate with the client-side and SOAP processor by the HTTP and HTTPS. 


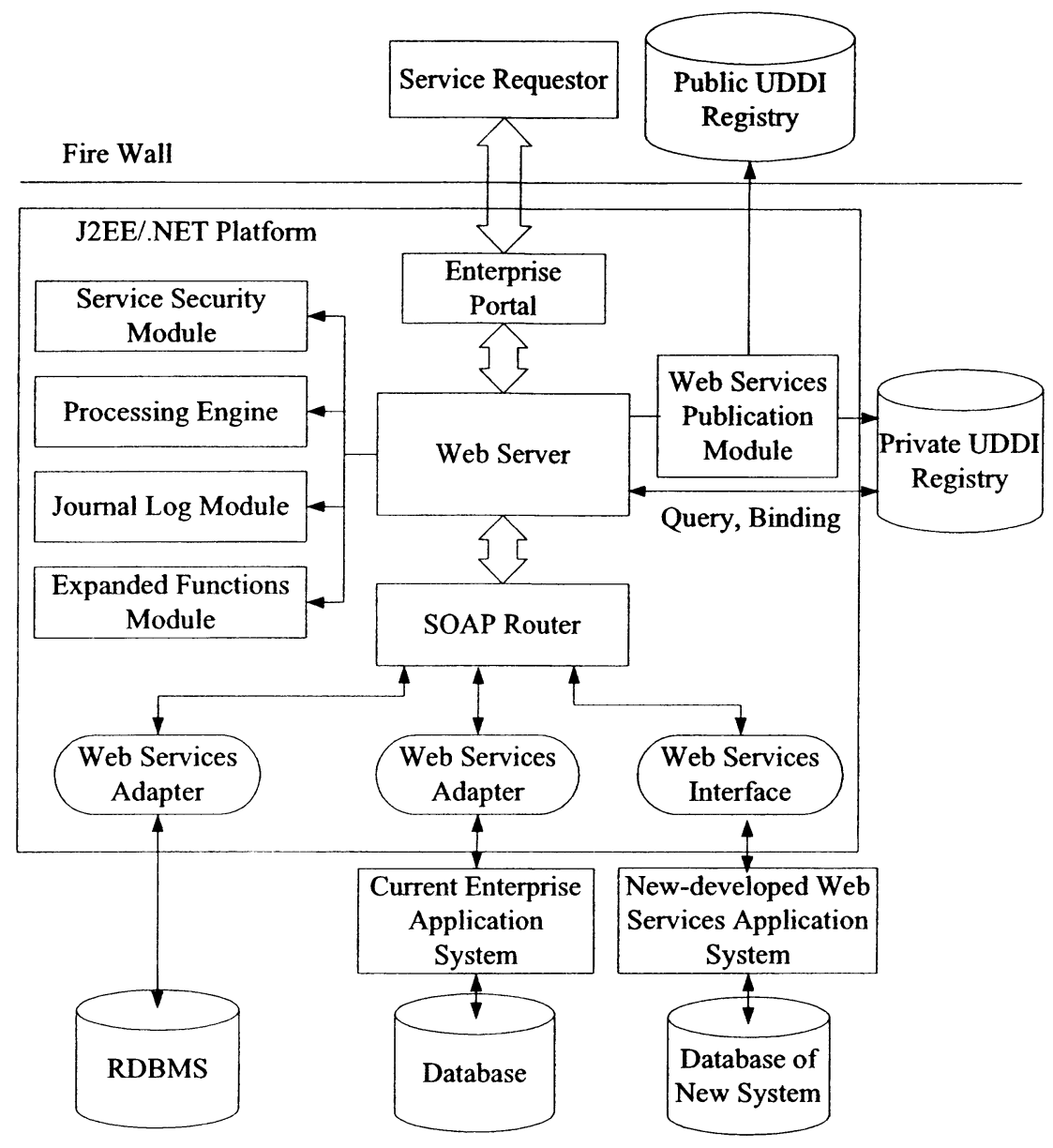

Figure 1. Web Services-based EAI Framework (WSEAI)

(4) Web Services Publication Module: Under the .NET platform, we could use the UDDI SDK programming to publish the Web Services to the private and public UDDI Registry in batch.

(5) UDDI registry: Public UDDI registry is open and could be used to realize the Inter-EAI; while the private UDDI registry shall be wrapped first and all the relevant Web Services Description Language (WSDL) shall be registered in the registry.

As the bridge of various application systems, the integration framework interface is coupled loosely, i.e., any application system could invoke its corresponding interface to connect to the system in a flexible and fast manner, and realizes the so-called "Plug \& Play" [3]. 
To integrate new application system, we need to describe its service with WSDL by Web Services adapter, and then publish the message to the UDDI registry through the SOAP. For intra-enterprise integration, the message shall be registered in the private UDDI registry. The message shall be published to the public UDDI registry for inter-enterprise integration, which is useful for the partner enterprises or the prospective partners to find and invoke the services and realize the inter-enterprise application system integration.

\section{APPLICATION OF WSEAI}

There is a Logistic Company in Dalian, Liaoning, China. The frame of its information system is shown as Figure 2.

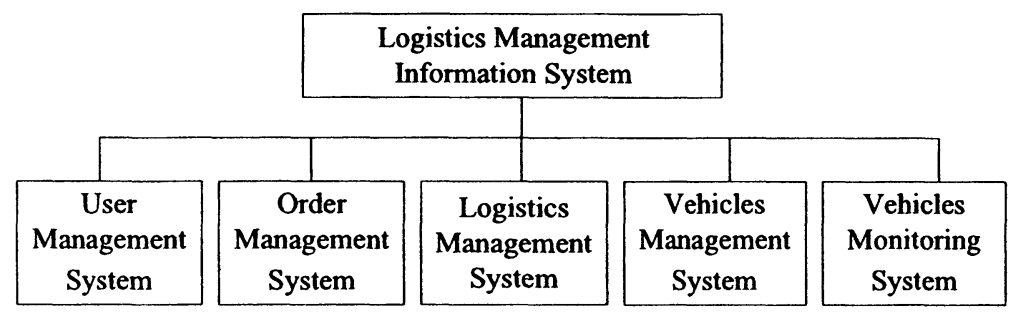

Figure 2. The Frame of Information System

According to Figure 2, the original information system of the logistics company has five sub-systems, in which the Vehicle Monitoring System is mainly used to monitor the transport vehicles by GPS. Almost without data communication with other systems, the Vehicle Monitoring System is relatively independent. The vehicle management department uses the GSM number to manage the vehicles. To facilitate the clients to follow the position of their goods, the company needs to establish a B/S vehicles monitoring system network, which we called WebGPS system. One of the main functions of the WebGPS is that after passing the authentication, the client could check the position of the transport vehicle by submitting the order number, and the position could be demonstrated in the electronic map on the Internet. It would be a waste of manpower and material resources if we develop a new WebGPS, which involves the sub-systems like User Management System, Order Management System and Logistics Management System, etc., and the redesign of the data flow. What's more, redevelopment of the monitoring module needs to use the GPS technology, serial port operation technology, GSM data communication protocol and GPS data decode, etc. But we could make full use of the original system by the thought of EAI. The following is an example of realizing the WSEAI in the WebGPS system

Based on the thought of EAI framework, we use Web Services to renew the original system, and expose the required functions of the original systems through the Web Services interface, which could be invoked by the WebGPS system, and the entire system framework of the .NET platform is as Figure 3: 


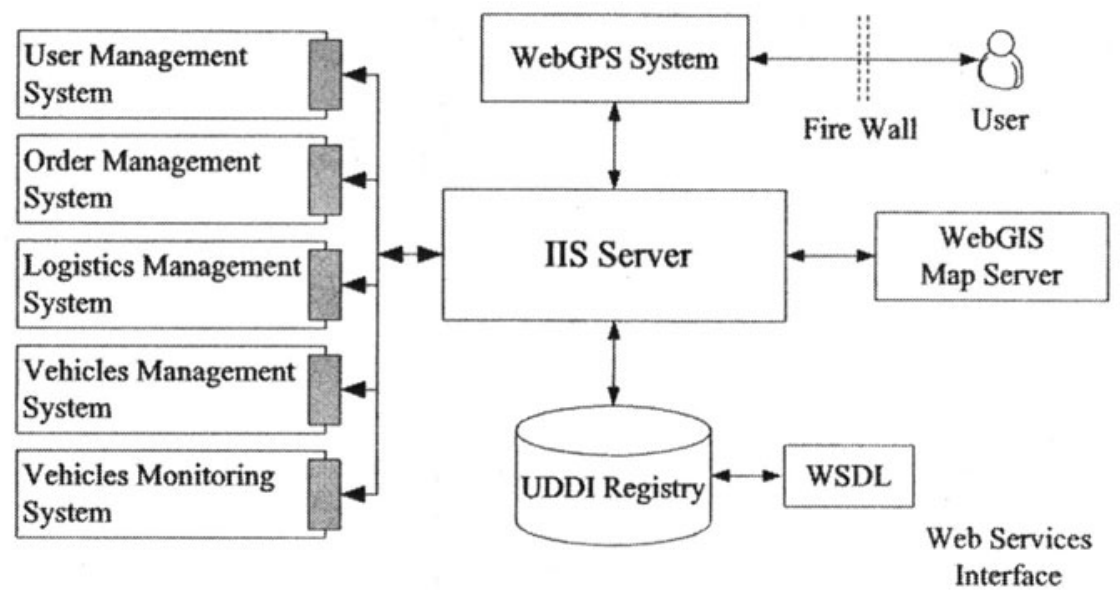

Figure 3. Web Services-based WebGPS Integration System

\section{SUMMARY}

In conclusion, compared with the traditional EAI solution, the application of the Web Services technology is more flexible and efficient. By wrapping the legacy systems, the original system is still independent. The wrapped interfaces are all in XML data format, and the service request and response is based on the SOAP protocol, which can easily realize the integration of the legacy systems and the newly developed Web Services application systems, etc. What's more, formed by a series of standards, the Web Services owns independent platform and language, which is more suitable for the heterogeneous platforms integration. In addition, the UDDI registry could help to realize the dynamic Web Services invocation, which has great advantage over the traditional static integration solutions.

\section{REFERENCES}

1. X. Chai and Y. Liang, Technology, Framework and Application of Web Services (Electronic and Industrial Press: Beijing, 2003).

2. V.N. Gudivada and J. Nandigam, Enterprise Application Integration Using Extensible Web Services, in Proc. of the IEEE International Conference on Web Services (ICWS'05) (2005), pp.41-48.

3. X. Chai. SOAP Technology and B2B Application Integration (2001). http://www.ibm.com/developerworks/cn/xml/soap/index.html 